\title{
Turbo Equalization Techniques Toward Robust PDM 16-QAM Optical Fiber Transmission
}

Arlunno, Valeria; Caballero Jambrina, Antonio; Borkowski, Robert; Saldaña Cercos, Silvia; Zibar, Darko; Larsen, Knud J.; Tafur Monroy, Idelfonso

Published in:

Journal of Optical Communications and Networking

Link to article, DOI:

$10.1364 / J O C N .6 .000204$

Publication date:

2014

Document Version

Publisher's PDF, also known as Version of record

Link back to DTU Orbit

Citation $(A P A)$ :

Arlunno, V., Caballero Jambrina, A., Borkowski, R., Saldaña Cercos, S., Zibar, D., Larsen, K. J., \& Tafur Monroy, I. (2014). Turbo Equalization Techniques Toward Robust PDM 16-QAM Optical Fiber Transmission. Journal of Optical Communications and Networking, 6(2), 204-214. https://doi.org/10.1364/JOCN.6.000204

\section{General rights}

Copyright and moral rights for the publications made accessible in the public portal are retained by the authors and/or other copyright owners and it is a condition of accessing publications that users recognise and abide by the legal requirements associated with these rights.

- Users may download and print one copy of any publication from the public portal for the purpose of private study or research.

- You may not further distribute the material or use it for any profit-making activity or commercial gain

- You may freely distribute the URL identifying the publication in the public portal 


\title{
Turbo Equalization Techniques Toward Robust PDM 16-QAM Optical Fiber Transmission
}

\author{
Valeria Arlunno, Antonio Caballero, Robert Borkowski, Silvia Saldaña Cercós, \\ Darko Zibar, Knud J. Larsen, and Idelfonso Tafur Monroy
}

\begin{abstract}
In this paper, we show numerically and experimentally that turbo equalization (TE) is an efficient technique to mitigate performance degradations stemming from optical fiber propagation effects in both optical fiber dispersion managed and unmanaged coherent detection links. The effectiveness of the proposed solution can be appreciated in both linear and nonlinear regimes for either scenario. We report on a system employing a polarization division multiplexing (PDM) 16-quadrature amplitude modulation (QAM) format for which we accomplish an increment in tolerance to link input power of up to $3 \mathrm{~dB}$ that represents a substantial improvement margin. The best bit error rate (BER) performances will therefore be guaranteed in a larger window, $6 \mathrm{~dB}$, of link input power thanks to the implemented TE scheme. Moreover, our proposed approach is also proven to effectively mitigate interchannel impairments from surrounding amplitude shift-keying interfering channels in a dispersion managed link achieving also in this case an increment in power tolerance of $3 \mathrm{~dB}$. Furthermore, in terms of BER performances, our proposed TE approach guarantees a gain of about a half order of magnitude at the best operational point. As TE can be included in the current coherent detection transceiver technologies and complement other equalization techniques, it has prospects for application in next-generation high-capacity and long-reach optical transmission links.
\end{abstract}

Index Terms-Coherent detection; Convolutional codes; Equalizers; Optical transmission; Signal processing algorithms; Turbo equalization.

\section{INTRODUCTION}

$\mathbf{H}$ igher-order modulation formats constitute an attractive solution toward capacity upgrade of the existing optical fiber infrastructure by exploiting their important feature of high spectral efficiency [1-5]. For example, polarization division multiplexing $(\mathrm{P} \overline{\mathrm{D}} \overline{\mathrm{M}})$ 16-quadrature amplitude modulation (QAM) is considered as a promising candidate for doubling the bit rate per channel with respect to PDM quadrature phase-shift keying (QPSK). In these transmission systems, linear fiber channel impairments, such as chromatic dispersion, group velocity dispersion,

Manuscript received May 2, 2013; revised December 18, 2013; accepted December 22, 2013; published January 31, 2014 (Doc. ID 189917).

The authors are with DTU Fotonik, Department of Photonics Engineering, Technical University of Denmark, Ørsteds Plads 343, 2800 Kgs. Lyngby, Denmark (e-mail: vaar@fotonik.dtu.dk).

http://dx.doi.org/10.1364/JOCN.6.000204 and polarization mode dispersion (PMD), can be effectively mitigated using digital linear signal processing techniques, as has been demonstrated for both QPSK and higher-order QAM signaling [6]. However, transmitter and receiver imperfections, not entirely mitigated by linear techniques, still need to be addressed. Linear processing algorithms are shown to be less effective for compensation of performance impairments stemming from optical fiber nonlinearities, such as self-phase modulation, four-wave mixing, cross-phase modulation, and laser phase noise. Additionally, in mixed modulation format transmission links, nonlinear impairments induced by coexisting $10 \mathrm{~Gb} / \mathrm{s}$ amplitude shift-keying (ASK) channels introduce a serious degradation to the overall system performance [7]. Moreover, when long transmission reach is targeted, higher power levels launched into the fiber might be required to guarantee extended reach; however, such high optical power level fiber transmission conditions enhance the generation of nonlinear optical fiber impairments.

Several techniques have been reported in the literature to mitigate for nonlinear optical fiber impairments, such as digital backpropagation, maximum likelihood sequence estimation (MLSE), use of radio frequency pilot, pre- and post-compensation, or expectation maximization [요뎌]. However, these methods suffer from complexity and depend on particular optical fiber transmission scenarios. Therefore there is a need for efficient equalization techniques to enhance the benefit of the aforementioned nonlinear techniques or to allow us to search for a new lower-complexity solution.

In this paper, we present a scheme based on turbo equalization (TE) equally effective in mitigating accumulated noise induced by link amplification and the effects induced by fiber nonlinearities. We realize a digital signal processing (DSP) structure based on convolutional code (CC), normalized least mean square (NLMS) equalization, and a soft-input soft-output (SISO) routine. TE routines have been proposed for direct detection optical communication systems in order to mitigate the effects of PMD $[15,16]$. In coherent detection optical fiber communications, until now, experimental demonstration of low-density paritycheck (LDPC) coded TE [17-19] has been presented for polarization multiplexed binary phase-shift keying (BPSK) and QPSK. More recently LDPC-coded TE has also been 
evaluated in electrical generated PDM orthogonal frequency division multiplexing (OFDM) 16-QAM for transoceanic transmission with large-core/ultra-low-loss fiber [20]. Even though LDPC codes can be very powerful, their implementation remains a challenge due to the complex structure of the decoder, and alternative works are looking for simplified low-complexity solutions [21].

We expand the state of the art by introducing TE for coherent optical links using an advanced modulation format, 16-QAM, including the transmission scenario with unmanaged and managed optical links with standard single-mode fiber (SSMF) and also in the presence of legacy channels in a $100 \mathrm{GHz}$ spectral grid. We perform experimental and numerical validation of the performance of our proposed TE for different fiber transmission lengths. We evaluate the performance both in the linear regime, where the performance is mostly degraded by chromatic dispersion and amplified spontaneous emission (ASE) noise introduced by the amplifier of the transmission link, and in the nonlinear regime, where nonlinearities are the dominant effects of performance degradation. Nevertheless further investigations should be realized to better evaluate the proposed scheme when specific nonlinear effects are targeted. Our TE approach features low complexity stemming from the use of a CC, and its ability to accomplish for each new decoding iteration a more accurate channel estimation. Concatenated Reed-Solomon (RS), Bose-ChaudhuriHocquenghem (BCH), and LDPC codes are employed as error correcting schemes in current optical communication products. In our case the employment of a simple and weaker code, i.e., CC, has been chosen to show the advantages of the TE routine and to avoid complexity challenges that solutions based on the other codes would present.

All the presented results in this paper have been obtained employing a PMD 16-QAM coherent detection system. We first implement a TE scheme in a simulation tool, and then we perform experimental validation. For completeness of results we study the effectiveness of our approach in a dispersion uncompensated link, through numerical investigations, and in a dispersion compensated link, through experimental investigations. Both experiment and simulation show that an increment in power tolerance up to $3 \mathrm{~dB}$ and a bit error rate (BER) gain of a half order of magnitude can be accomplished, confirming the efficiency of our approach for both scenarios. Additionally we perform experimental validation for PDM 16-QAM transmission surrounded by neighbor $10 \mathrm{~Gb} / \mathrm{s}$ ASK channels in a $100 \mathrm{GHz}$ spectral grid, a situation in which the transmission reach is severely distorted by cross nonlinear impairments due to the interactions with the copropagating ASK channels. A total increment in power tolerance of up to $3 \mathrm{~dB}$ is also accomplished in this case. Both $\mathrm{CC}$ and the equalizer contribute in the TE routine to achieve the obtained improvements.

\section{Turbo Equalization}

Inspired by turbo codes, TE was first introduced to combat intersymbol interference effects in digital communication systems [22]. A TE system consists of an iterative joint equalization and decoding technique that can achieve impressive performance gains in terms of signal-to-noise ratio (SNR) for communication systems.

Figure 1 shows the pre-encoder and receiver structure for TE employed in this work. Random generated data 1000 bits long are created with offline processing and encoded by a CC with rate $R=1 / 2$ and 16 states trellis. The 2000 coded bits are then interleaved to suppress burst errors and sent to the optical modulator at the transmitter in the physical layer. CCs have up to now only been incompletely explored for optical communications. The drawback of such codes in optical transmission is their high coding overhead compared to high-rate block codes, such as RS codes and LDPC codes. On the other hand, CC codes present attractive features such as simple and straightforward encoding and hard and soft decision decoding algorithms. The large overhead can be alleviated by using CC codes together with bandwidth-efficient modulation. Similar performance to RS codes has been obtained, with the benefit of simpler encoding electronics [23]. In this work we present a simple CC not only for the features presented, but also for convenience purposes in demonstrating its benefits and the advantages of TE. However, a more complex code can be designed and evaluated in the future.

On the receiver side, the received signal after optical transmission and detection is then processed offline. After a 16-QAM DSP receiver [12], the received data are fed into a SISO TE structure. A NLMS adaptive equalizer is applied due to the unknown nature of the transmission channel. We optimize the number of taps and the step to obtain the best performance. Soft information on all the coded bits in the form of log-likelihood ratios (LLRs) is provided by the demapper. A nongray 16-QAM mapping results due to the typical setup configuration on optical systems based on independent binary electronics, requiring a specific

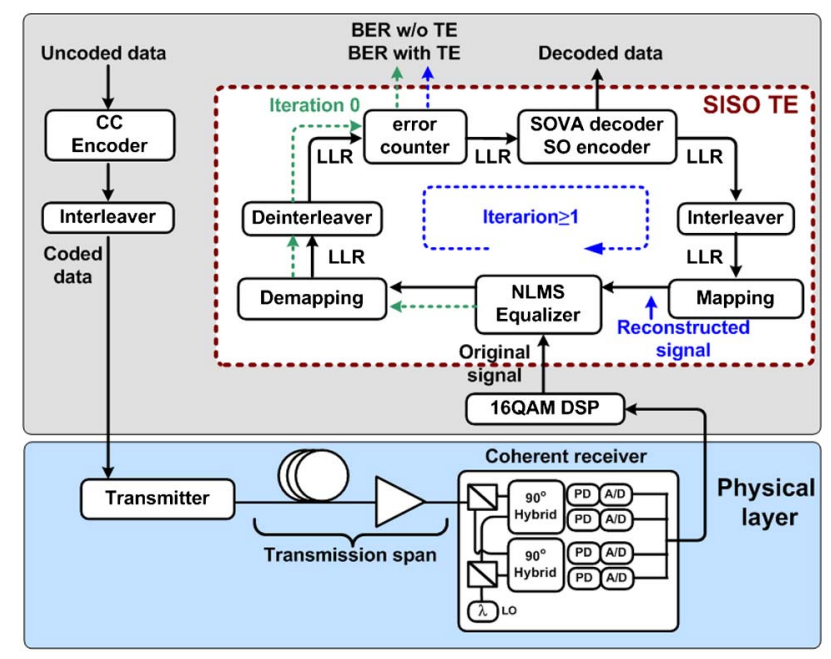

Fig. 1. Pre-encoding and receiver structure of the SISO turbo equalizer algorithm. CC, convolutional code; LO, local oscillator; $\mathrm{PD}$, photodiode; A/D, analog-to-digital converter; DSP, digital signal processing; NLMS, normalized least mean square; LLR, log-likelihood ratio; SOVA, soft-output Viterbi algorithm; SO, soft output; SISO, soft-input soft-output; TE, turbo equalization. 
demapper implementation. In this mapping, not all the neighbor symbols differ in only one bit, thus requiring a specific LLR calculation. For more details regarding mapping and LLR calculation, please refer to Appendix A. Thereafter we apply a deinterleaver process, symmetrical to the one employed at the transmitter side. An error counter will then give us the performance of the system in terms of BER. The performance without TE, later presented in this work, is calculated at this point and is also called "iteration 0" and "w/o TE," to highlight that no complete iteration has been accomplished and we are exiting the loop. Moving forward with the TE iteration, we perform decoding using a soft-output Viterbi algorithm (SOVA) binary decoder with 64 states to obtain soft estimation of both information and parity bits. We then re-encode the obtained decoded version of our signal to create a reconstructed signal. After passing through an interleaving and mapping stage, to reproduce the original constellation, new soft estimates of the original transmitted symbols are obtained. We then realize an NLMS equalization process using the information of the original and reconstructed signal. Demapping, deinterleaving and decoding can be performed as for the first iteration. In every iteration we evaluate the BER at the error counter stage. In this work the results presented as "with TE" represent just the first complete iteration of the TE routine. The use of a nongray mapping also influences the reconstruction part of the loop and therefore could originate difficulties in the realization of more iteration, as explained in Appendix $\underline{A}$.

\section{Simulation And Experimental Setup}

\section{A. Simulation Setup}

The setup used for the numerical simulations is presented in Fig. 2. The numerical setup is composed of three main blocks: 4-pulse amplitude modulation (PAM) generation and transmitter, link transmission, coherent receiver, and DSP modules. At the transmitter side the output of a $100 \mathrm{kHz}$ linewidth laser is passed through an optical I/Q modulator driven by two four-level PAM electrical signals to generate the optical 16-QAM single polarization (SP) signal. The 4-PAM module consists of an encoding process, signal mapping, upsampling, pulse shaping, digital-toanalog conversion, attenuators, and electrical amplifiers. The baud rate is kept at 28 Gbaud. The orthogonal polarization, $Y$, is created with the same procedure. The two signals are then combined with a polarization beam combiner (PBC) to create a PDM 16-QAM signal. In the link transmission block the PDM 16-QAM signal is amplified and launched into the fiber span link. The transmission link consists of spans of SSMF of $100 \mathrm{~km}$ length. Three link lengths of 1000,1500 , and $2000 \mathrm{~km}$, corresponding to a number of spans equal to 10,15 , and 20 , respectively, are evaluated. For the SSMF we have the following fiber parameters: $\alpha_{\mathrm{smf}}=0.2 \mathrm{~dB} / \mathrm{km}, D_{\mathrm{smf}}=17 \mathrm{ps} / \mathrm{nm} / \mathrm{km}$, and the nonlinear coefficient is $\gamma_{\mathrm{sm}}=1.3 \mathrm{~W}^{-1} \mathrm{~km}^{-1}$. Erbiumdoped fiber amplifier (EDFA) amplification is employed after each span, compensating fully the fiber losses. At the receiver side we apply coherent detection and DSP modules. The received PDM 16-QAM signal is coherently detected in a 90 deg optical hybrid, photodetected, and sampled at twice the baud rate by the analog-to-digital converter. We consider a $100 \mathrm{kHz}$ linewidth laser as the local oscillator (LO). The DSP module includes a digital filter, $\mathrm{CD}$ compensation, radius directed equalization (RDE), polarization demultiplexing, and frequency and carrier phase recovery [12]. After demodulation the signal is fed into the SISO TE structure presented earlier.

\section{B. Experimental Setup}

The setup used for the experimental evaluations for the PDM 16-QAM coherent optical system is presented in Fig. 3. It is composed of three main blocks: 16-QAM generation and transmitter, link transmission, and coherent receiver and DSP modules. A 2000 bit data frame encoded by a CC is applied to the system. The data are pre-encoded offline and loaded into the memory of a programmable pulse pattern generator (PPG). The data outputs at 10 Gbaud are delayed to guarantee decorrelation, subsequently combined to create a four-level electrical signal,

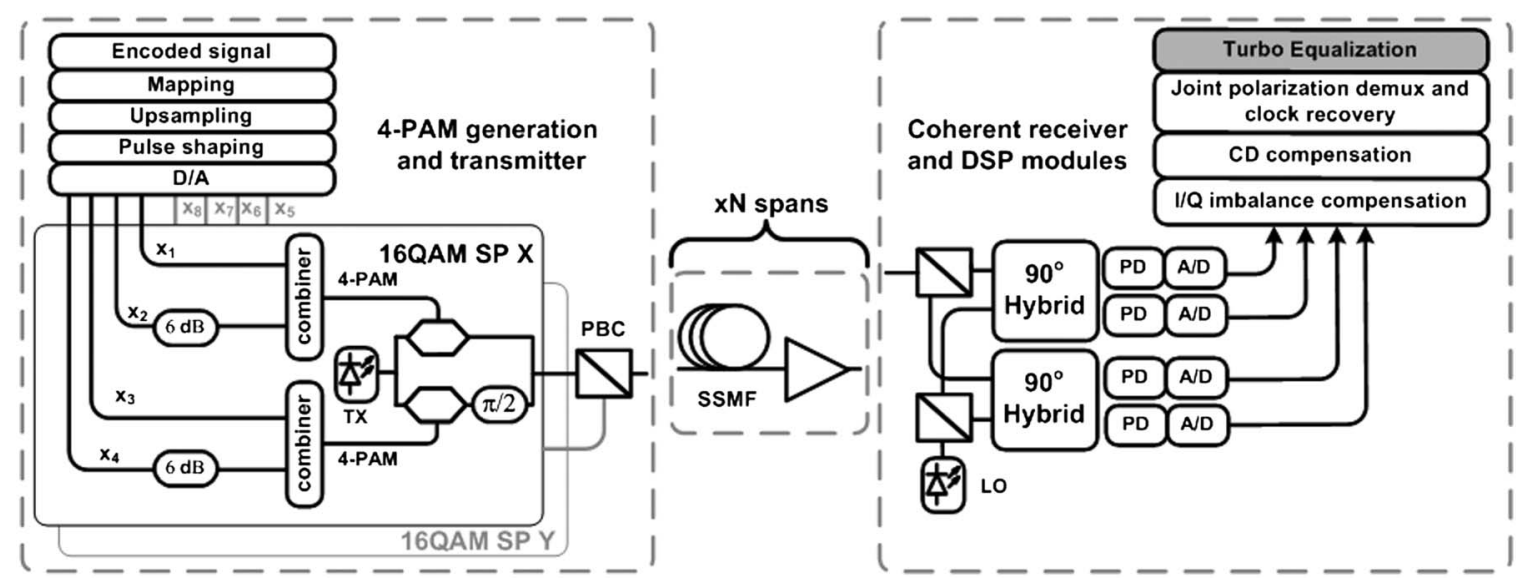

Fig. 2. Simulation setup. D/A, digital-to-analog converter; SP, single polarization; PBC, polarization beam combiner; PD, photodetector; $\mathrm{A} / \mathrm{D}$, analog-to-digital converter. 


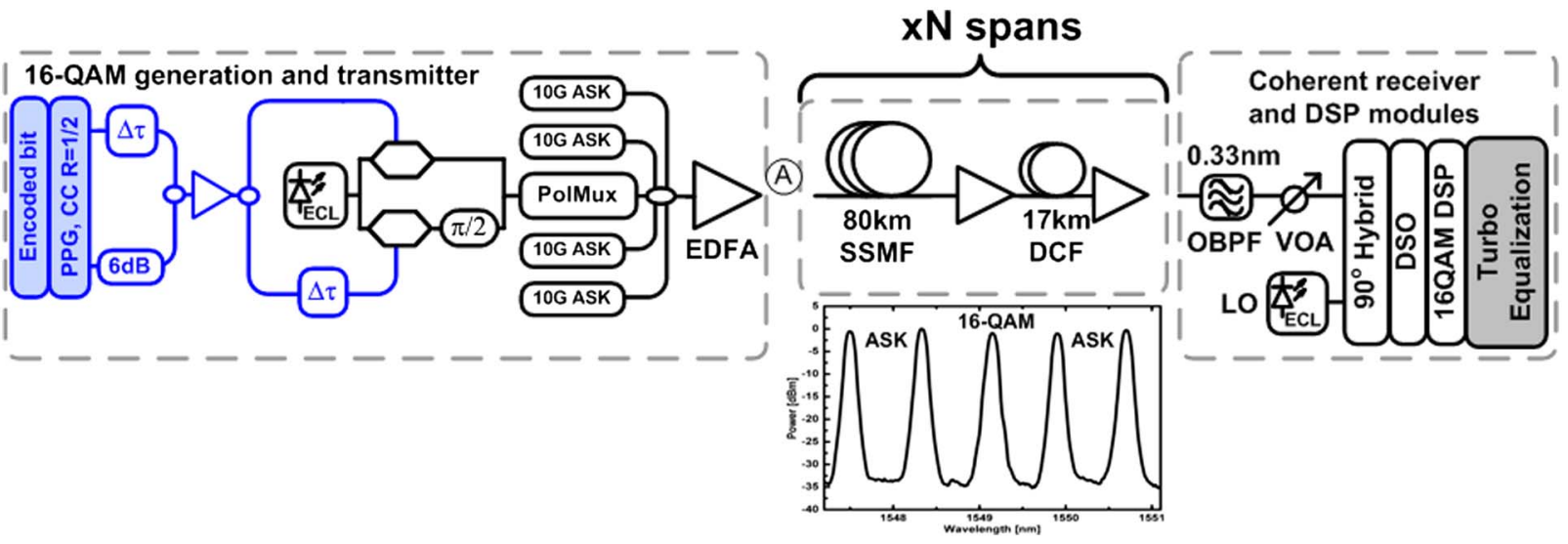

Fig. 3. Experiment setup. PPG, pulse pattern generator; CC, convolutional code; ECL, external cavity laser; EDFA, erbium-doped fiber amplifier; A, launch power measurement point; OBPF, optical bandpass filter; VOA, variable optical attenuator.

amplified, and split into two branches to be fed to an I/Q optical modulator. An external cavity laser (ECL) with a linewidth of $100 \mathrm{kHz}$ is employed at the transmitter. The optical 16-QAM signal is then sent to a polarization multiplexing stage to generate the PDM 16-QAM signals. The experimental evaluations are reported for a baud rate of $10 \mathrm{Gbaud}$ and a bit rate of $80 \mathrm{~Gb} / \mathrm{s}$. Four $10 \mathrm{G}$ transponders are then multiplexed with the 16-QAM signal to emulate the interfering channels, which have been placed symmetrically around the central signal with a spacing of $100 \mathrm{GHz}$. The spectrum of the signal with the neighboring four legacy ASK channels is presented as an inset of Fig. 3. In the center is placed the PDM 16-QAM encoded signal, surrounded by two ASK channels on both sides in a $100 \mathrm{GHz}$ spacing grid. An EDFA is used to adjust the launch power to the link, including a precompensation of the dispersion equivalent to $40 \mathrm{~km}$ of SSMF. The transmission link block consists of spans of SSMF of $80 \mathrm{~km}$ length and dispersion compensated fiber (DCF) of $17 \mathrm{~km}$ length. EDFA amplification is used for span loss compensation. Three link lengths 240, 400, and $640 \mathrm{~km}$, corresponding to a number of spans equal to 3,5 , and 8 , respectively, are evaluated. The input power to the link, measured at point A, is varied between -4 and $4 \mathrm{dBm}$ with a granularity of $1 \mathrm{~dB}$. At the receiver side, we apply coherent detection and DSP modules. We employ an ECL with $100 \mathrm{kHz}$ linewidth as the LO and a variable optical attenuator at the signal input to maintain a constant ratio between the signal received and the LO. A $40 \mathrm{GSa} / \mathrm{s}$ sampling rate digital signal oscilloscope (DSO) with $13 \mathrm{GHz}$ bandwidth is employed to sample the received signals, and then offline signal processing is performed. The PDM 16-QAM DSP-based receiver earlier presented for the numerical validation case, with exception of the CD compensation module not present for the compensated link, is then applied followed by the SISO TE structure presented above.

\section{Metodology}

The measured curve for the BER as a function of the link input power, presented in Figs. 3, 5, 6, and 7, and the measured curve for the BER as a function of the lasers' linewidth, presented in Fig. 4, show results without (w/o) $\mathrm{TE}$ and with TE. The first case, when no TE is employed, corresponds to "iteration 0." After demodulating the signal, we apply the hard decision to obtain the stream of bits that forms the received signal. This stream is then directly compared to the one used at the transmitter side in the BER counter block. When TE is applied, we employ the SISO TE structure, using therefore the LLR information of the received signals and making a full iteration. We will then exit the loop at the BER counter stage, as shown in Fig. 1, before starting a new iteration. In both cases, iteration 0 and iteration 1 , the error counter is placed before the soft decoder; therefore the results reflect the performance of the original bits, w/o TE, and of the reconstructed bits, with $\mathrm{TE}$, and not the performance of the CC. This also means that the results reported all have the same line rate, and baud rate, chosen at the transmission side, while the decoded results, not reported in this work, would show better performances but with a net rate equal to half the line rate, due to the rate chosen for the CC.

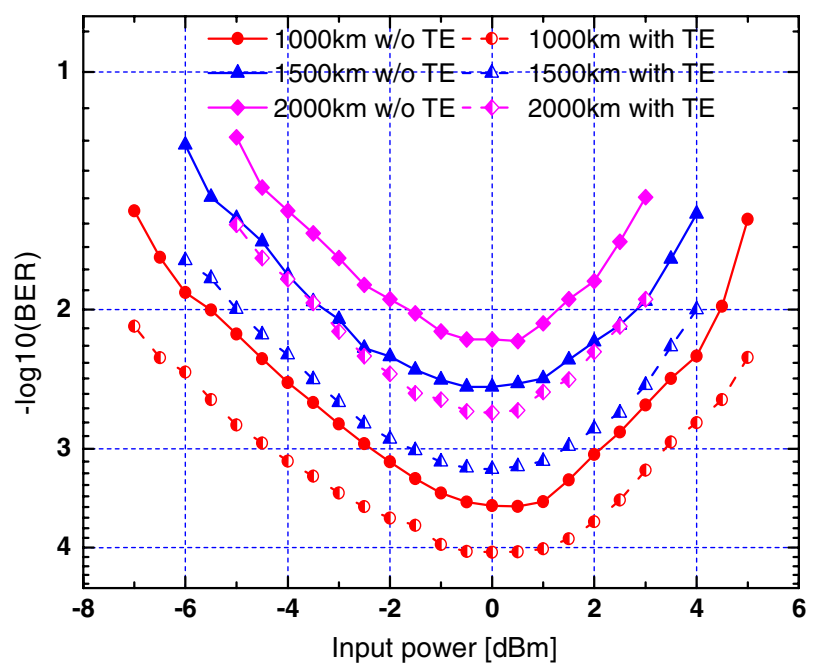

Fig. 4. BER as a function of link input power to the transmission span with and without TE for 1000, 1500, and $2000 \mathrm{~km}$ uncompensated SSMF transmission. 


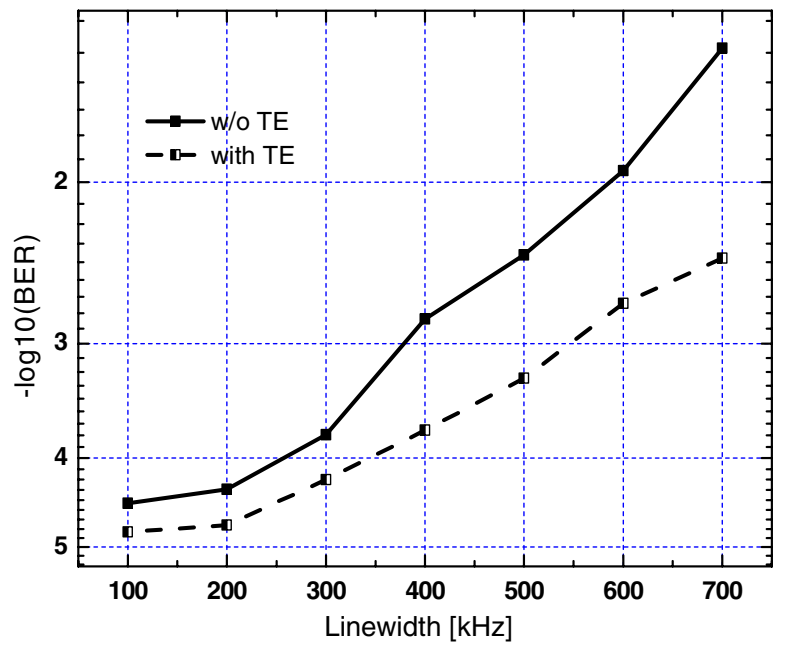

Fig. 5. BER as a function of transmitter and local oscillator laser linewidth for back-to-back with a fixed OSNR value of $25 \mathrm{~dB}$.

For the numerical evaluation of the PDM 16-QAM signal we have created three different realizations, while for the experimental results we have evaluated three different traces of the transmitted signal. That holds for all the results presented and for all the transmission distances considered. In all of them, for both polarizations and for all four bits that constitute the 16-QAM signal, 52 frames of the encoded original data were retrieved. This gives an amount of processed data equal to $\sim 2.5 \times 10^{6}$ bits, which guarantees sufficient bits for error count of the BER values reported for both numerical and experimental results.

\section{Performances Over Uncompensated LinK}

We first evaluate the performance for the dispersion uncompensated link scenario, through numerical investigations. We study the effectiveness of our approach for

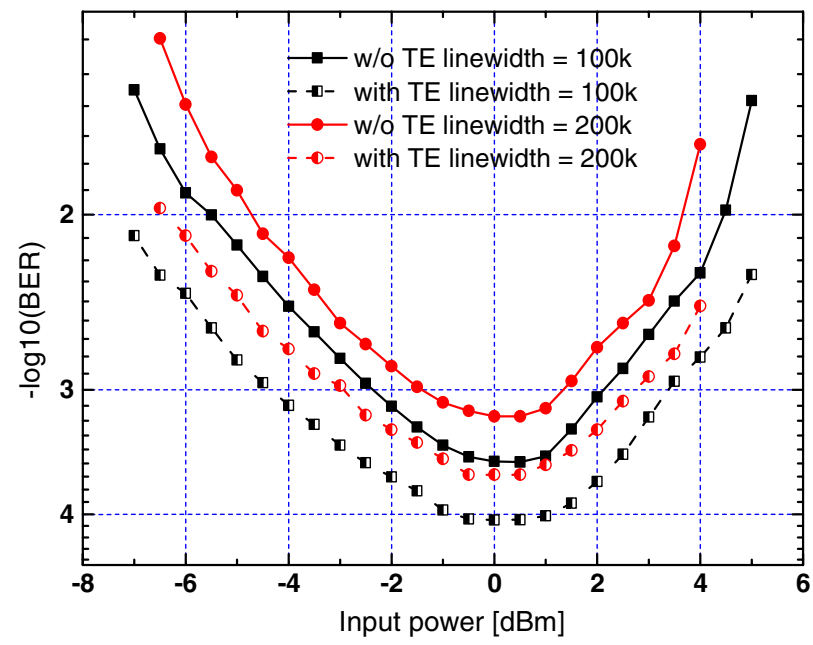

Fig. 6. BER as a function of link input power to the transmission span with and without TE for 100 and $200 \mathrm{kHz}$ linewidth for $1000 \mathrm{~km}$ uncompensated SSMF transmission.

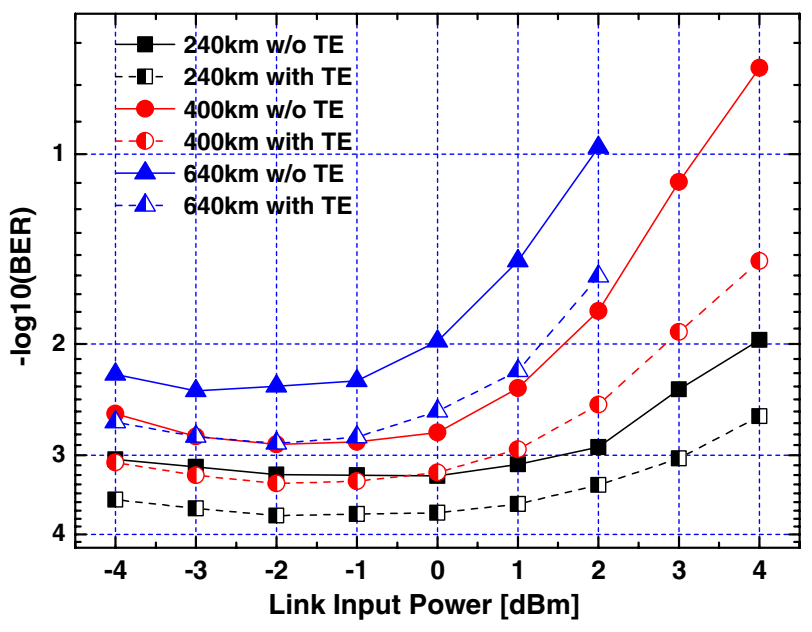

Fig. 7. Performance of single channel. BER as a function of link input power to the transmission span with and without TE for 240 , 400, and $640 \mathrm{~km}$ compensated link.

different transmission lengths. We then demonstrate that similar performance obtained can be expected with a more commercial laser with higher laser linewidth through numerical investigations of back-to-back and $1000 \mathrm{~km}$ total link length scenario.

We consider a transmission link consisting of spans of SSMF of $100 \mathrm{~km}$ length. The 16-QAM DSP modules block includes CD compensation, polarization demultiplexing, frequency, and carrier recovery. For the case in which TE is not used, the so-called iteration 0, hard decision BER evaluation is applied. For the case when TE is used, the SISO TE structure explained above is employed after demodulation. We employ a NLMS adaptive equalizer; the number of taps and step size is kept constant throughout all the numerical investigations. A SOVA, based on the soft information provided by the LLR and with 64 states, is used as the decoder. The results of the BER performances as a function of link input power for three different distances are shown in Fig. 4. A total link reach of 1000, 1500, and $2000 \mathrm{~km}$, corresponding to a number of spans of 10,15 , and 20 , respectively, has been investigated. We can evaluate the results both on link input power accomplished tolerance and on BER performance gain. It is observed from Fig. 4 that by employing the TE, we accomplish an increment in tolerance to link input power of up to $2 \mathrm{~dB}$. If we consider as an example the case of $1000 \mathrm{~km}$ transmission, the best operational point is obtained for a link input power equal to $0 \mathrm{dBm}$. The same BER performance can be obtained for a link input power to $2 \mathrm{dBm}$ when the TE routine is applied, resulting in the mentioned total increment in tolerance of $2 \mathrm{~dB}$. The same BER performance can be obtained for $-2 \mathrm{dBm}$, and therefore the best operational point BER performance can now be obtained over a window of link input power of $4 \mathrm{~dB}$, from -2 to $2 \mathrm{dBm}$. In terms of BER performance, our proposed TE approach guarantees a gain of about a half order of magnitude at the best operational point. It should also be noted that this algorithm can extend the maximum total length by $500 \mathrm{~km}$, which corresponds to five spans. We can notice that the results 
obtained employing the TE scheme in a $2000 \mathrm{~km}$ link outperform the results of a $1500 \mathrm{~km}$ length link when no TE is applied.

We then demonstrate that the performances obtained are not related to the laser linewidth value. First we evaluate a back-to-back case, no transmission, and we vary the value of the linewidth parameter of both the transmitter and the LO laser from 100 to $700 \mathrm{kHz}$ with a granularity of $100 \mathrm{kHz}$. In Fig. 5, we numerically evaluate the BER performances as a function of the value for laser linewidth for a fixed optical signal-to-noise ratio (OSNR) of $25 \mathrm{~dB}$. The results obtained show that our TE routine has similar behavior for all the laser linewidth values considered and can therefore be implemented with commercial distributed feedback lasers. It should also be noted that the TE routine is more effective in the low-performance region, from 400 to $700 \mathrm{kHz}$, where an improvement of almost one order of magnitude can be seen.

Next we demonstrate the independence from the laser linewidth also in the transmission scenario. We consider uncompensated link transmission with $1000 \mathrm{~km}$ of total distance length, and we vary the value of the linewidth parameter of both the transmitter and LO lasers from 100 to $200 \mathrm{kHz}$. The results are shown in Fig. 6. In this case it is observed that by employing the TE, we accomplish an increment in tolerance to link input power of up to $2 \mathrm{~dB}$, and the best operational point BER performance can now be obtained over a window of link input power of $4 \mathrm{~dB}$. Figure 6 also shows that by employing the $\mathrm{TE}$ routine, the results obtained with a linewidth value of $200 \mathrm{kHz}$ outperform the ones with $100 \mathrm{kHz}$ when no TE has been applied. This means that the use of our proposed solution can relax the requirements in terms of laser linewidth value for the transmitter and receiver.

\section{EXPERIMEntal Result for Compensated Optical Fiber Transmission LinK}

We evaluate the dispersion compensated link scenario through experimental investigations. We study the effectiveness of our approach for different transmission lengths; we then evaluate its effects in the presence of copropagating interfering ASK channels.

We consider a dispersion managed transmission link consisting of spans of SSMF of $80 \mathrm{~km}$ length. The dispersion effect is compensated optically by $17 \mathrm{~km}$ of DCF. The 16-QAM DSP modules block includes polarization multiplexing, frequency, and carrier recovery. For the case in which TE is not used, the so-called iteration 0 , hard decision BER evaluation is applied. For the case in which TE is used, the SISO TE structure explained above is employed after demodulation. We employ a NLMS adaptive equalizer; we optimize the number of taps and the step for all the realizations. A SOVA, based on the soft information provided by the LLR and with 64 states, is used as the decoder.

\section{A. Performances of Single Channel Transmission}

First, we evaluate the BER performances obtained through experimental investigations for a single channel transmission scenario. In Fig. 7 the BER performance is plotted as a function of the link input power. We evaluate three link transmission lengths: 240,400 , and $640 \mathrm{~km}$, corresponding to 3,5 , and 8 spans, respectively. We vary the value of the link input power from -4 to $+4 \mathrm{dBm}$ with a granularity of $1 \mathrm{dBm}$. We then evaluate the results both on link input power accomplished tolerance and on BER performance gain. It is observed that by employing the TE, we accomplish an increment in tolerance to link input power of up to $3 \mathrm{~dB}$, and the best operational point BER performance can now be obtained over a window of link input power of $6 \mathrm{~dB}$. The use of the $\mathrm{CC}$ can provide a maximum improvement of $\sim 5 \mathrm{~dB}$ in the loop, which is seen in the following way. A performance of $10^{-3}$ after the soft encoder corresponds to $\sim 6 \mathrm{~dB}$ of SNR. Before the soft encoder we have a BER of $(1 / 7) \times 10^{-3}$ (since the generator has 7 ones) corresponding to an $\mathrm{Eb} / \mathrm{N} 0$ of $\sim 4 \mathrm{~dB}$. To obtain the aforementioned BER, $\sim 1 \mathrm{~dB}$ in entrance to the SOVA block is required. Thus a maximum gain of $\sim 5 \mathrm{~dB}$ is provided by the presence of the $\mathrm{CC}$ in the loop. The $6 \mathrm{~dB}$ increment in the launch power window is therefore provided by the use of the complete TE scheme. In terms of BER performances our proposed $\mathrm{TE}$ approach also guarantees a gain of about a half order of magnitude at the best operational point. It should also be noted that the results obtained using the TE routine for 400 and $640 \mathrm{~km}$ have similar performances to the results obtained with 240 and $400 \mathrm{~km}$, respectively, when no TE has been applied. For all the transmission lengths, we observe that larger improvement in terms of BER is achieved when the original performances are worse, in this case between 2 and $4 \mathrm{dBm}$ of link input power, where the performance degradation is also dominated by nonlinear effects.

\section{B. Performances With Interfering Channels}

In Fig. 8, the BER performance as a function of the link input power is plotted for a multichannel transmission scenario employing PDM 16-QAM and $10 \mathrm{~Gb} / \mathrm{s}$ ASK surrounding channels in a $100 \mathrm{GHz}$ grid. The 16-QAM signal is placed in the center, while the four interfering channels are placed two on each side. The spectrum is reported as an inset of the experimental setup in Fig. 3. As for the single channel transmission scenario we evaluate three link transmission lengths, 240,400 , and $640 \mathrm{~km}$, and we vary the value of the link input power from -4 to $+4 \mathrm{dBm}$ with a granularity of $1 \mathrm{dBm}$. It is observed that also in this case by employing the TE, we accomplish an increment in tolerance to link input power of up to $3 \mathrm{~dB}$, and the best operational point BER performance can now be obtained over a window of link input power of $6 \mathrm{~dB}$. In terms of BER performances, our proposed TE approach guarantees a gain of about a half order of magnitude at the best operational point. It should also be noted that the results obtained using the TE routine for 


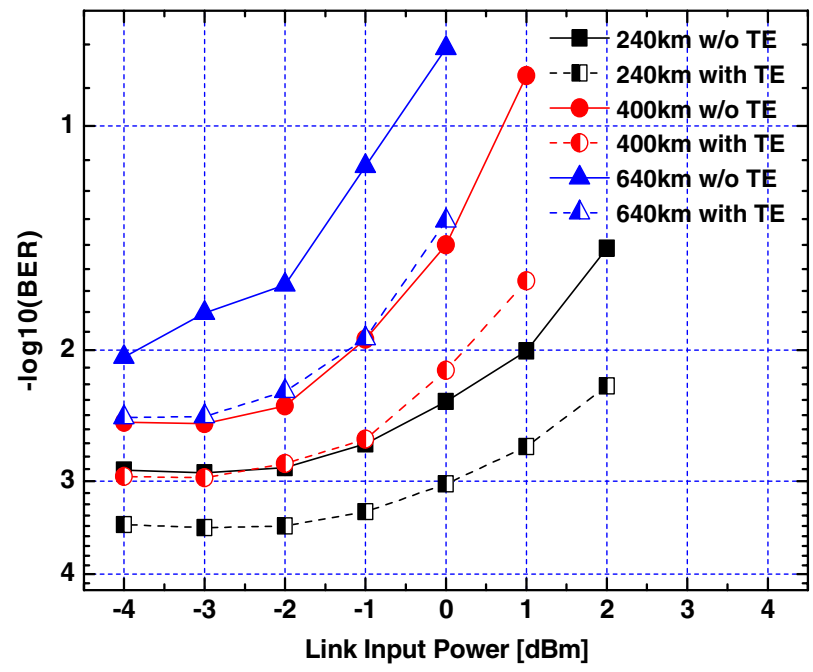

Fig. 8. Performance with interfering OOK channels. BER as a function of link input power to the transmission span with and without TE for 240, 400, and $640 \mathrm{~km}$ compensated link.

400 and $640 \mathrm{~km}$ show performance similar to the results obtained with 240 and $400 \mathrm{~km}$, respectively, when no TE has been applied. For all the transmission lengths, we observe that larger improvement in terms of BER is achieved for higher link input power, between 2 and $4 \mathrm{dBm}$, which is the region where the performance degradation is strongly dominated by nonlinear effects. If we consider, for example, $400 \mathrm{~km}$ transmission at $+1 \mathrm{dBm}$ link input power, we achieve an improvement of almost one order of magnitude in terms of BER performance.

Comparing this result with the single channel reported above, we notice that the total performance is affected by the presence of the ASK interfering channels, which cause a penalty of $3 \mathrm{~dB}$ in terms of input power. As an example, it can be pointed out that similar performance for $240 \mathrm{~km}$ and $3 \mathrm{~dB}$ of input power obtained with a single channel is now reported for the same distance with $0 \mathrm{~dB}$ of input power in the presence of ASK interfering channels.

\section{COMPARISON}

The BER performance has been evaluated for three different situations: uncompensated link transmission, through numerical simulations, and compensated link transmission, through experimental investigations, for both single and multichannel scenarios. The performances reported in Figs. 4, 7, and 8 have been obtained for the same sets of link input powers, from -4 to $+4 \mathrm{dBm}$ with a granularity of $1 \mathrm{dBm}$. Therefore we can compare the above-mentioned results in terms of gain system tolerance, which are reported in Table I. Power tolerance, in Table I, represents the accomplished tolerance to link input power obtained through the TE routine. From Table I, we observe that a $2 \mathrm{~dB}$ power increment in tolerance is guaranteed for all the numerical evaluations with an unmanaged transmission link. A maximum increment of $3 \mathrm{~dB}$ is obtained for the $1500 \mathrm{~km}$ transmission case. In the case of the dispersion managed link, investigated through experimental validation, we have evaluated single channel and multichannel transmission with interfering ASK channels, reported as $100 \mathrm{GHz}$ in Table I. For both of these cases and for all the transmission link lengths considered, TE results in a reported increment in power tolerance of $3 \mathrm{~dB}$. Similar to what we obtain for numerical validations, link transmission distances longer than the ones considered in this experimental validation should result in smaller increments. The column labeled "window" represents the sets of link input power for which the best operational point BER performance can be obtained. From Figs. 7 and 8 we can analyze only the right half of the tolerance, $3 \mathrm{~dB}$ in all cases, but the results should present the same properties as the numerical investigations, in which case the tolerance on the left side was equal to that on the right side. $\Delta$ BER represents the gain in terms of BER obtained by applying TE for the best operational values. We notice that an improvement of about a half order of magnitude has been obtained for numerical investigation. If we consider $1500 \mathrm{~km}$, at the best operational point the system presents a BER performance of $3.1 \times 10^{-3}$ without TE and of $6.8 \times$ $10^{-4}$ after the TE routine, for a total $\Delta$ BER of $6.9 \mathrm{~dB}$. This value represents the numerical evaluation of the BER improvement in terms of order of magnitude. Since one order of magnitude would correspond to $10 \mathrm{~dB}$, the results obtained confirm an improvement greater than a half order of magnitude. For the experimental validation we also obtain an improvement of almost a half order of magnitude for all the cases considered. The performance improvement is also reported in terms of the $Q$ factor.

TABLE I

Summary of Transmission Results

\begin{tabular}{|c|c|c|c|c|c|}
\hline & Distance $[\mathrm{km}]$ & Increment Tolerance $[\mathrm{dB}]$ & Window $[\mathrm{dB}]$ & $\Delta \mathrm{BER}[\mathrm{dB}]$ & $Q[\mathrm{~dB}]$ \\
\hline \multirow[t]{3}{*}{ Numerical single channel } & 1000 & 2.5 & 5 & 5.1 & 0.75 \\
\hline & 1500 & 3 & 6 & 6.8 & 1.36 \\
\hline & 2000 & 2 & 4 & 5.2 & 1.30 \\
\hline \multirow[t]{3}{*}{ Experiment single channel } & 240 & 3 & 6 & 4.6 & 0.75 \\
\hline & 400 & 3 & 6 & 4.5 & 0.81 \\
\hline & 640 & 3 & 6 & 5.2 & 1.01 \\
\hline \multirow[t]{3}{*}{ Experiment $100 \mathrm{GHz}$} & 240 & 3 & 6 & 5.4 & 0.97 \\
\hline & 400 & 3 & 6 & 4.7 & 0.97 \\
\hline & 640 & 3 & 6 & 4.6 & 1.17 \\
\hline
\end{tabular}




\section{ConcLuSION}

We have reported the impact of the TE structure for mitigation of transmission impairments in a coherently detected PDM 16-QAM system. A CC has been employed at the transmitter side, while at the receiver the investigated TE is based on a NLMS as equalizer and on a SOVA as a decoder. We observe from our studies that TE is effective in mitigating fiber transmission impairments in a single channel PDM 16-QAM. We have accomplished up to $3 \mathrm{~dB}$ of increment in power tolerance and about a half order of magnitude BER gain for both unmanaged and managed transmission links. The first scenario has been evaluated by numerical investigations, the latter by experimental validation. The use of a TE routine can therefore also improve the maximum total and span length; $500 \mathrm{~km}$ longer transmission has been obtained in the numerical validation case. On the other hand, the benefit of this method can be addressed to relax the optical component, such as the laser linewidth; evaluation of the effect of the transmitter and receiver lasers' linewidth has been realized for a $1000 \mathrm{~km}$ unmanaged SSMF transmission realized by numerical validation. Additionally our proposed solution is proven to be effective in the transmission scenario where performance and reach are severely distorted by cross nonlinear impairments due to the interactions with the copropagating ASK channels in a $100 \mathrm{GHz}$ spectral grid. An increment in system tolerance of $3 \mathrm{~dB}$ is also reported in this case. Both the $\mathrm{CC}$ and equalizer in the TE loop contribute to the achieved improvements.

The results show the potential of the proposed TE routine for promising high-speed, high-capacity optical networking, such as PDM 16-QAM, over unmanaged and managed systems based on SSMF. Further improvement and some future research directions of the results could be (i) the use of gray mapping, (ii) the use of more powerful codes, and (iii) concatenation of the $\mathrm{CC}$, and therefore of the TE scheme, with high-rate block codes.

\section{APPENDIX A}

In this appendix we show that the nongray mapping results in a specific calculation of the LLR values. In Fig. 9 two different mappings are shown: on the left side the gray mapping, and on the right side the nongray 16-QAM mapping resulting from the typical setup configuration of optical systems based on binary electronics. In Fig. 9 the value of the first two bits according to the region they belong to is also shown, for both mappings. As we can see, in the gray mapping design, if we move from left to right, only one bit of the two is changed every time we pass from one region to another. On the other side, in the nongray mapping, this feature is not present. We can notice that moving from region 2 to region 3 requires changing both bits at the same time. This fact also influences the LLR calculation.

The LLR calculation is realized for all four bits that constitute the 16-QAM signal and is based on the formulation reported in [24]. We indicate the four bits as $b_{\mathrm{Re}, k} b_{\mathrm{Re}, k} b_{\mathrm{Im}, k} b_{\mathrm{Im}, k}$ and $k=0,1$. The first two bits are

\begin{tabular}{|c|c|c|c|c|c|c|c|}
\hline \multicolumn{4}{|c|}{ Gray mapping } & \multicolumn{4}{|c|}{ Non-Gray mapping } \\
\hline$\stackrel{\circ}{0010}$ & $\stackrel{\circ}{0110}$ & $\stackrel{\circ}{1110}$ & $\stackrel{\circ}{1010}$ & 울 & $\stackrel{\circ}{0111}$ & $\stackrel{0}{1011}$ & $\stackrel{\circ}{1111}$ \\
\hline$\stackrel{\circ}{0011}$ & $\stackrel{\circ}{0111}$ & $\stackrel{\circ}{1111}$ & $\stackrel{\circ}{1011}$ & $\begin{array}{c}0 \\
0010\end{array}$ & $\stackrel{\circ}{0110}$ & $\stackrel{\circ}{1010}$ & $\stackrel{\circ}{1110}$ \\
\hline 0001 & 0101 & $\begin{array}{c}\circ \\
1101\end{array}$ & 1001 & 0001 & $0 \stackrel{\circ}{0101}$ & $\stackrel{\circ}{1001}$ & $\stackrel{\circ}{1101}$ \\
\hline 00000 & $0 \stackrel{\circ}{0100}$ & 1100 & $\stackrel{\circ}{1000}$ & 0000 & $0 \stackrel{\circ}{0100}$ & $\stackrel{\circ}{1000}$ & $\stackrel{\circ}{1100}$ \\
\hline$b_{0}=0$ & $b_{0}=0$ & $b_{0}=1$ & $b_{0}=1$ & $b_{0}=0$ & $b_{0}=0$ & $b_{0}=1$ & $b_{0}=1$ \\
\hline (1) & (2) & (3) & (4) & (1) & (2) & (3) & (4) \\
\hline $\mathbf{b}_{1}=0$ & $b_{1}=1$ & $\mathbf{b}_{1}=1$ & $b_{1}=0$ & $b_{1}=0$ & $\mathbf{b}_{1}=1$ & $\mathbf{b}_{1}=\mathbf{0}$ & $\mathbf{b}_{1}=1$ \\
\hline (1) & (2) & (3) & (4) & (1) & (2) & (3) & (4) \\
\hline
\end{tabular}

Fig. 9. Gray, left side, and nongray, right side, mapping employed in the system.

derived from the real part of the signal, while the last two are from the imaginary part of the signal. The idea is demapping the received signal into soft bits that have the same sign as provided by a hard detector and whose absolute value represents the reliability of the decision. The optimum hard decision on bit $b_{\mathrm{Re}, k}$ is given by the rule

$$
\begin{aligned}
\hat{b}_{\mathrm{Re}, k} & =\beta \quad \text { if } P\left[b_{\mathrm{Re}, k}=\beta \mid r\right] \\
>P\left[b_{\mathrm{Re}, k}\right. & =(1-\beta) \mid r], \quad \beta=0,1,
\end{aligned}
$$

where $r$ represents the received signal

$$
r=G_{\mathrm{ch}} \cdot a+w,
$$

where $G_{\mathrm{ch}}$ is the channel frequency response and $w$ is the complex additive white Gaussian noise (AWGN). If we set $\beta=1$, we can rewrite Eq. (A1) as

$$
\hat{b}_{\mathrm{Re}, k}=1, \quad \text { if } \log \frac{P\left(r \mid b_{\mathrm{Re}, k}=1\right)}{P\left(r \mid b_{\mathrm{Re}, k}=0\right)}>0 .
$$

When we consider $k=0$ we obtain

$$
\frac{P\left(r \mid b_{\mathrm{Re}, 0}=1\right)}{P\left(r \mid b_{\mathrm{Re}, 0}=0\right)}=\frac{e^{\frac{-\left(\left(_{\mathrm{Re}}-1\right)^{2}\right.}{2 \sigma^{2}}}+e^{\frac{-\left(\mathrm{V}_{\mathrm{Re}}-3\right)^{2}}{2 \sigma^{2}}}}{e^{\frac{-\left(y_{\mathrm{Re}}+1\right)^{2}}{2 \sigma^{2}}}+e^{\frac{-\left(y_{\mathrm{Re}}+3\right)^{2}}{2 \sigma^{2}}}} .
$$

The formulation in Eq. (A4) has been obtained considering that when $b_{\mathrm{Re}, 0}=0$ the real part of the 16-QAM can only assume negative values, -3 or -1 , and when $b_{\mathrm{Re}, 0}=1$ the real part of the 16-QAM can only assume positive values, +1 or +3 . Therefore the LLR formulation for $b_{\mathrm{Re}, 0}$ is the same as reported in [24] 


$$
\operatorname{LLR}_{\mathrm{Re}, 0}=\left\{\begin{array}{lll}
\frac{4}{\sigma^{2}}\left(y_{\mathrm{Re}}[i]+1\right), & y_{\mathrm{Re}}[i]<-2, & \text { region } 1 \\
\frac{2}{\sigma^{2}} y_{\mathrm{Re}}[i], & \left|y_{\mathrm{Re}}[i]\right| \leq 2, & \text { regions } 2 \& 3 . \\
\frac{4}{\sigma^{2}}\left(y_{\mathrm{Re}}[i]-1\right), & y_{\operatorname{Re}}[i]>+2, & \text { region } 4
\end{array}\right.
$$

Similarly to what is obtained for $b_{\mathrm{Re}, 0}$, we can obtain the formulations for $b_{\mathrm{Re}, 1}$ :

$$
\frac{P\left(r \mid b_{\mathrm{Re}, 1}=1\right)}{P\left(r \mid b_{\mathrm{Re}, 1}=0\right)}=\frac{e^{\frac{-\left(\mathrm{Y}_{\mathrm{R}}-3\right)^{2}}{2 \sigma^{2}}}+e^{\frac{-\left(\mathrm{g}_{\mathrm{R}}+1\right)^{2}}{2 \sigma^{2}}}}{e^{\frac{-\left(\mathrm{Y}_{\mathrm{R}}-1\right)^{2}}{2 \sigma^{2}}}+e^{\frac{-\left(\mathrm{Y}_{\mathrm{R}}+3\right)^{2}}{2 \sigma^{2}}}} .
$$

The LLR formulation in Eq. (A6) has been obtained considering that when $b_{\mathrm{Re}, 1}=0$ the real part of the 16-QAM can only assume values -3 or +1 , and when $b_{\text {Re, } 1}=1$ the real part of the 16-QAM can only assume values -1 or +3 .

We now consider $b_{\mathrm{Re}, 1}$. In region 2 , when $-2 \leq y_{\mathrm{re}}<0$, we can assume that the relative contribution by constellation point +3 in the numerator [Eq. (A8)] can be ignored. Therefore the likelihood ratio reduces to

$$
\frac{P\left(r \mid b_{\mathrm{Re}, 1}=1\right)}{P\left(r \mid b_{\mathrm{Re}, 1}=0\right)} \approx \frac{e^{\frac{-\left(\mathrm{Re}_{\mathrm{Re}}+1\right)^{2}}{2 \sigma^{2}}}}{e^{\frac{-\left(\mathrm{G}_{\mathrm{Re}}+3\right)^{2}}{2 \sigma^{2}}}+e^{\frac{-\left(\mathrm{V}_{\mathrm{Re}}-1\right)^{2}}{2 \sigma^{2}}}} .
$$

Expressing Eq. (A7) in logarithm form and considering negligible the added term $e^{\left((-8) / 2 \sigma^{2}\right)}$, we obtain the LLR formula for $b_{\mathrm{Re}, 1}$ in region 2 as

$$
\operatorname{LLR}\left(b_{\mathrm{Re}, 1}\right)_{\operatorname{reg} 2}=\frac{2}{\sigma^{2}}\left(-y_{\mathrm{Re}}\right) .
$$

Considering all four regions for $b_{\mathrm{Re}, 0}$ and $b_{\mathrm{Re}, 1}$ we obtain the LLR formulas summarized in Table II.

The LLR formulas calculation for $b_{\mathrm{Im}, 0}$ and $b_{\mathrm{Im}, 1}$ follows the same rules as for $b_{\mathrm{Re}, 0}$ and $b_{\mathrm{Re}, 1}$. The results obtained are therefore the same, but on the imaginary part of the signal. We assume that all the clusters have the same variance and we therefore remove the factor $2 / \sigma^{2}$ from all the LLR formulas. A study on the different variances of the 16 clusters can lead to a more precise calculation of the LLR and therefore to better performance.

The remapping block, placed after the SOVA decoder in the TE routine, works with the inverse formula reported in Table II. The four LLR values are then used to reconstruct the real and imaginary signals. It should be noted that only

TABLE II

LLR FORMULAS FOR $b_{\mathrm{Re}, 0}$ AND $b_{\mathrm{Re}, 1}$

\begin{tabular}{cccc}
\hline & & $\operatorname{LLR}\left(b_{\mathrm{Re}, 0}\right)$ & $\operatorname{LLR}\left(b_{\mathrm{Re}, 1}\right)$ \\
\hline Region 1 & $y<-2$ & $\left(4 / \sigma^{2}\right)\left(y_{\mathrm{Re}}+1\right)$ & $\left(2 / \sigma^{2}\right)\left(y_{\mathrm{Re}}+2\right)$ \\
Region 2 & $-2 \leq y<0$ & $\left(2 / \sigma^{2}\right) y_{\mathrm{Re}}$ & $\left(2 / \sigma^{2}\right)\left(-y_{\mathrm{Re}}\right)$ \\
Region 3 & $0 \leq y<+2$ & $\left(2 / \sigma^{2}\right) y_{\mathrm{Re}}$ & $\left(2 / \sigma^{2}\right)\left(-y_{\mathrm{Re}}\right)$ \\
Region 4 & $y \geq+2$ & $\left(4 / \sigma^{2}\right)\left(y_{\mathrm{Re}}-1\right)$ & $\left(2 / \sigma^{2}\right)\left(y_{\mathrm{Re}}-2\right)$ \\
\hline
\end{tabular}

one of the LLR values between $b_{\mathrm{Re}, 0}$ and $b_{\mathrm{Re}, 1}$ is necessary to reconstruct the real part, and only one between $b_{\mathrm{Im}, 0}$ and $b_{\text {Im, } 1}$ to reconstruct the imaginary part. Due to this fact and to the mapping scheme, the reconstruction process may present jumps beyond the nearest cluster of the constellation. For example, let us consider the complex signal $+1.3+1.3 j$. It will be demapped into 1010 , and the LLRs are calculated with the formulas presented. Let us now assume that we have a decoder/re-encoder error, and the new bits are 0010. In this case we can notice that the corrections of the error forced us to move from region 3 to region 1 for the real part of the signal, and therefore not to the nearest clusters.

\section{REFERENCES}

[1] D. van den Borne, V. Sleiffer, M. S. Alfiad, and S. L. Jansen, "Towards 400G and beyond: How to design the next generation of ultra-high capacity transmission systems," in Proc. of OptoElectronics and Communications Conf., July 2011, pp. 429-432.

[2] P. J. Winzer, "Beyond 100G Ethernet," IEEE Commun. Mag. vol. 48 , no. 7 , pp. 26-30, July 2010.

[3] X. Zhou and L. E. Nelson, "400G WDM transmission on the $50 \mathrm{GHz}$ grid for future optical networks," J. Lightwave Technol., vol. 30, no. 24, pp. 3779-3792, Dec. 2012.

[4] J. Yu, Z. Dong, H. C. Chien, Z. Jia, X. Li, D. Huo, M. Gunkel, P. Wagner, H. Mayer, and A. Schippel, "Transmission of 200G PDM-CSRZ-QPSK and PDM-16QAM with a SE of $4 \mathrm{~b} / \mathrm{s} / \mathrm{Hz}$," J. Lightwave Technol., vol. 31, no. 4, pp. 515-522, Feb. 2013.

[5] P. J. Winzer, "High-spectral-efficiency optical modulation formats," J. Lightwave Technol., vol. 30, no. 24, pp. 3824-3835, Dec. 2012.

[6] S. J. Savory, "Digital coherent optical receivers: Algorithms and subsystems," IEEE J. Sel. Top. Quantum Electron., vol. 16, no. 5, pp. 1164-1179, Sept.-Oct. 2010.

[7] T. Inoue, E. Mateo, F. Yaman, T. Wang, Y. Inada, T. Ogata, and Y. Aoki, "Low complexity nonlinearity compensation for 100G DP-QPSK transmission over legacy NZ-DSF link with OOK channels," in 38th European Conf. on Optical Communication, Sept. 2012, paper Mo.1.C.5.

[8] E. Ip and J. Kahn, "Compensation of dispersion and nonlinear impairments using digital backpropagation," J. Lightwave Technol., vol. 26, no. 20, pp. 3416-3425, Oct. 2008.

[9] A. Lau and J. Kahn, "Signal design and detection in presence of nonlinear phase noise," J. Lightwave Technol., vol. 25, no. 10, pp. 3008-3016, Oct. 2007.

[10] Z. Tao, L. Dou, W. Yan, L. Li, T. Hoshida, and J. C. Rasmussen, "Multiplier-free intrachannel nonlinearity compensating algorithm operating at symbol rate," J. Lightwave Technol., vol. 29, no. 17, pp. 2570-2576, Sept. 2011.

[11] N. Stojanovic, Y. Huang, F. N. Hauske, Y. Fang, M. Chen, C. Xie, and Q. Xiong, "MLSE-based nonlinearity mitigation for WDM $112 \mathrm{Gbit} / \mathrm{s}$ PDM-QPSK transmissions with digital coherent receiver," in Proc. of Optical Fiber Communication Conf., 2011, paper OTu3C.5.

[12] D. Zibar, O. Winther, N. Franceschi, R. Borkowski, A. Caballero, V. Arlunno, M. N. Schmidt, N. Guerrero Gonzalez, B. Mao, Y. Ye, K. J. Larsen, and I. Tafur Monroy, "Nonlinear impairment compensation using expectation maximization for PDM 16-QAM systems," Opt. Express, vol. 20, no. 26, pp. B181-B196, 2012. 
[13] E. Ip, P. Ji, E. Mateo, Y. Huang, L. Xu, D. Qian, N. Bai, and T. Wang, "100G and beyond transmission technologies for evolving optical networks and relevant physical-layer issues," Proc. IEEE, vol. 100, no. 5, pp. 1065-1078, 2012.

[14] D. Rafique, J. Zhao, and A. D. Ellis, "Digital back-propagation for spectrally efficient WDM $112 \mathrm{Gbit} / \mathrm{s}$ PM m-ary QAM transmission," Opt. Express, vol. 19, no. 6, pp. 5219-5224, 2011.

[15] M. Jäger, T. Rankl, J. Speidel, H. Bülow, and F. Buchali, "Performance of turbo equalizers for optical PMD channel," $J$. Lightwave Technol., vol 24, no. 3, pp. 1226-1236, Mar. 2006.

[16] H. F. Haunstein, T. Schorr, A. Zottmann, W. Sauer-Greff, and R. Urbansky, "Performance comparison of MLSE and iterative equalization in FEC systems for PMD channels with respect to implementation complexity," J. Lightwave Technol., vol. 24, no. 11, pp. 4047-4054, Nov. 2006.

[17] I. B. Djordjevic, L. L. Minkov, and H. G. Batshon, "Mitigation of linear and nonlinear impairments in high-speed optical networks by using LDPC-coded turbo equalization," IEEE J. Sel. Areas Commun., vol. 26, no. 6, pp. 73-83, Aug. 2008.

[18] L. L. Minkov, I. B. Djordjevic, L. Xu, and T. Wang, "PMD compensation in polarization-multiplexed multilevel modulations by turbo equalization," IEEE Photon. Technol. Lett., vol. 21 , no. 23 , pp. 1773-1775, Dec. 2009.

[19] L. L. Minkov, I. B. Djordjevic, L. Xu, and T. Wang, "Suppression of fiber nonlinearities and PMD in coded-modulation schemes with coherent detection by using turbo equalization," J. Opt. Commun. Netw., vol. 1, no. 6, pp. 555-564, Nov. 2009.

[20] S. Zhang, Y. Zhang, M. Huang, F. Yaman, E. Mateo, D. Qian, L. Xu, Y. Shao, and I. B. Djordjevic, "Transoceanic transmission of $40 \times 117.6 \mathrm{~Gb} / \mathrm{s}$ PDM-OFDM-16QAM over hybrid large-core/ultralow-loss fiber," J. Lightwave Technol., vol. 31, no. 4, pp. 498-505, Feb. 2013.

[21] M. N. Sakib, V. Mahalingam, W. J. Gross, and O. LiboironLadouceur, "Optical front-end for soft-decision LDPC codes in optical communication systems," J. Opt. Commun. Netw., vol. 3, no. 6, pp. 533-541, June 2011.

[22] C. Douillard, M. Jézéquel, C. Berrou, A. Picart, P. Didier, and A. Glavieux, "Iterative correction of intersymbol interference: Turbo-equalization," Eur. Trans. Telecommun., vol. 6, no. 5, pp. 507-511, 1995.

[23] T. Wuth, E. Agrell, M. Karlsson, and M. Sköld, "Fiber communications using convolutional coding and bandwith-efficient modulation," Opt. Express, vol. 14, no. 2, pp. 542-555, 2006.

[24] F. Tosato and P. Bisaglia, "Simplified soft-output demapper for binary interleaved COFDM with application to HIPERLAN/2," in Proc. of IEEE Int. Conf. on Communications, 2002, p. 664 .

Valeria Arlunno was born in Gattinara, Italy, in 1984. She received her B.Sc. in 2006 and M.Sc. from the Politecnico di Torino, Torino, Italy, in 2009. In 2009 she joined the Optcom group at Politecnico di Torino as a research assistant. She is currently pursuing a Ph.D. in optical communications engineering at DTU Fotonik, Technical University of Denmark, with the Metro-Access and Short Range Systems research group of the Department of Photonics Engineering. Her research interests are in the areas of coherent optical communications and digital signal processing.

Antonio Caballero was born in 1985 in Zaragoza, Spain. He received B.Sc. and M.Sc. degrees in telecommunications engineering from Centro Politécnico Superior, Zaragoza, Spain, in 2008.
He received his $\mathrm{Ph} . \mathrm{D}$. in optical communications engineering at DTU Fotonik, Technical University of Denmark, in September 2011, with a thesis on high-capacity radio-over-fiber links. He was a visiting researcher at The Photonics and Networking Research Laboratory at Stanford University from February to June 2010, under supervision of Prof. Leonid G. Kazovsky. He is currently a postdoctoral researcher at DTU Fotonik. His research interests are in the area of coherent optical communications as well as radio-over-fiber links.

Robert Borkowski was born in Lodz, Poland, in 1987. He received the M.Sc. degree in telecommunications from the Technical University of Denmark in 2011 and the M.Sc. degree in electronics and telecommunications from the Technical University of Lodz in 2012. He is now working toward his Ph.D. degree in the field of optical communications in the Metro-Access and Short Range Systems group at DTU Fotonik, Department of Photonics Engineering, Technical University of Denmark. He is involved in the EU FP7 project CHRON (Cognitive Heterogeneous Reconfigurable Optical Networks). His research interests are in the areas of optical performance monitoring, coherent transmission systems, and digital signal processing for fiber-optic communications.

Silvia Saldaña Cercós was born in Barcelona, Spain, in 1985. She received her B.Sc. in 2009 from Universitat Politècnica de Catalunya, Spain, and her M.Sc. from DTU Fotonik, Technical University of Denmark, in 2011. She is currently pursuing a Ph.D. in optical communications engineering at DTU Fotonik, Technical University of Denmark, with the Metro-Access and Short Range Systems research group of the Department of Photonics Engineering. Her research interests are in the areas of energy efficiency in coherent optical communications and digital signal processing.

Darko Zibar was born in Belgrade, in the former Yugoslavia, on September 9, 1978. He received the M.Sc. degree in telecommunication and the Ph.D. degree in optical communications from the Technical University of Denmark, Lyngby, Denmark, in 2004 and 2007, respectively. He was a visiting researcher with the Optoelectronic Research Group, University of California, Santa Barbara, during January-August 2006 and in January 2008, where he was involved in coherent receivers for phase-modulated analog optical links. From February to July 2009, he was a visiting researcher with Nokia-Siemens Networks, where he worked on $112 \mathrm{Gbit} / \mathrm{s}$ polarization multiplexed systems. He is currently an associate professor at DTU Fotonik, Technical University of Denmark. His research interests include coherent optical communication, with an emphasis on digital demodulation and compensation techniques.

Dr. Zibar is the recipient of the Best Student Paper Award at the IEEE Microwave Photonics Conference 2006, the Villum Kann Rasmussen Postdoctoral Research Grant in 2007, and the Villum Foundation Young Investigator Program in 2011.

Knud J. Larsen received M.S.E.E. and Ph.D. degrees from the Technical University of Denmark, Lyngby, Denmark. He was a senior engineer and project manager at Ericsson Signal Systems, where he was involved in design and implementation of communication systems and traffic control systems. Since 1986, he has been a professor at the Technical University of Denmark (previously the Institute of Telecommunication, now the Department of Photonics), teaching and performing research in communication theory and coding. He has worked extensively on studies of error correction for the European Space Agency and is currently involved in projects related to optical transmission.

Dr. Larsen together with four colleagues received the Information Theory Society Paper Award in 1991 for a paper on algebraic geometry codes. 
Idelfonso Tafur Monroy is currently a professor and the head of the Metro-Access and Short Range Systems group of the Department of Photonics Engineering at the Technical University of Denmark. He graduated from the Bonch-Bruevitch Institute of Communications, St. Petersburg, Russia, in 1992, where he received an M.Sc. degree in multichannel telecommunications. In 1996 he received a Technology Licentiate degree in telecommunications theory from the Royal Institute of Technology, Stockholm, Sweden. In the same year he joined the Electrical Engineering Department of the Eindhoven University of Technology, The Netherlands, where he earned a Ph.D. degree in 1999 and worked as an assistant professor until 2006. He has participated in several European research framework projects in photonic technologies and their applications to communication systems and networks. At the moment he is involved in the ICT European projects GigaWaM and EURO-FOS and is the technical coordinator of the CHRON project. His research interests are in hybrid opticalwireless communication systems, high-capacity optical fiber communications, digital signal processing for optical transceivers for baseband and radio-over-fiber links, application of nanophotonic technologies in the metropolitan and access segments of optical networks, and short range optical-wireless communication links. 